\title{
WDM: An Energy-Efficient Multi-hop Routing Algorithm for Wireless Sensor Networks
}

\author{
Zheng Zengwei ${ }^{1,2}$, Wu Zhaohui ${ }^{1}$, Lin Huaizhong ${ }^{1}$, and Zheng Kougen ${ }^{1}$ \\ ${ }^{1}$ College of Computer Science, Zhejiang University, \\ 310027 Hangzhou, China \\ \{Zhengzw, Wzh, Linhz, Zkg\}@Cs.zju.edu.cn \\ ${ }^{2}$ City College, Zhejiang University, \\ 310015 Hangzhou, China \\ Zhengzw@zucc.edu.cn
}

\begin{abstract}
As a new technique, one characteristic of wireless sensor networks (WSNs) is their limited system lifetime. Therefore, it is more important to save energy and proportion energy consumption. This paper presents a weightdirected based multi-hop routing algorithm for WSNs. This algorithm can transfer data quickly to goal sensor node using the directional information and RWVs (route weight value) of sensor nodes as well as balance energy consumption of all sensor nodes. Detailed simulations of sensor network environments indicate that this algorithm improves energy efficiency and proportions energy consumption of all sensor nodes to extend network system lifetime, and routes data quickly in comparison to the flooding algorithm.
\end{abstract}

\section{Introduction}

Recent advances in micro-electro-mechanical systems (MEMS) technology, wireless communications, and digital electronics have enabled the development of wireless sensor networks (WSNs) consisting of a large number of low-cost, low-power, multifunctional sensor nodes which are small in size and communicate untethered in short distances. As a new technique of implementing ubiquitous computing [1][2], WSNs can be used in many aspects in the coming future, such as military battlefield surveillance, patient health monitoring [3], bio-environment monitoring [4] and industrial process control. Since the sensor nodes are often inaccessible in most applications and supported by battery, the lifetime of a wireless sensor network depends on the lifetime of the power resources of the sensor nodes. Hence, WSNs have one characteristic different from traditional Ad Hoc networks, i.e. their system lifetime is limited.

The characteristic of limited lifetime indicates that energy is a very scarce resource for the sensor systems and requires a new sensor network with low energy consumption in order to extend the lifetime of the sensors for the duration of a particular mission. Since the main goal in designing conventional Ad hoc networks is providing high quality of service, conventional wireless network protocols for Ad Hoc networks are not well suitable for WSNs. Furthermore, requirements of designing routing algorithm for WSNs is different from those for traditional Ad Hoc networks, i.e. it needs more energy savings. Therefore, it is significant to study new routing algorithms for WSNs. 
As multi-hop routing shortens communication distance, short transmission range will reduce packet collisions, enable channel reuse in different regions of a wireless sensor network, lower energy consumption of sensor nodes, and prolong the lifetime of sensor nodes. Hence, multi-hop routing idea is suitable for WSNs. Existing multihop routing algorithms, such as flooding, gossiping [7] and directed diffusion [5][6], have several advantages and disadvantages. Flooding can perform simply, route data quickly, and it does not require costly topology maintenance and complex route discovery algorithms. However, when it broadcasts packets to its neighbors, implosion problem will be generated, and a large number of data flow and subsequently channel congestion, and communication overheads may happen. Therefore, its energyefficiency is wondrously low. A derivation of flooding is gossiping [7] in which nodes do not broadcast but send the incoming packets to a randomly selected neighbor. Although the implosion phenomenon can be avoided by the copy of a message at any sensor node, it takes long time to propagate the message to goal sensor node (sink node) because it does not use the directional information to route data quickly to objective node. Directed diffusion [5][6] is a data-centric and applicationaware routing protocol. All sensor nodes in the directed diffusion-based network are application-aware, which enables diffusion to achieve energy savings by selecting empirically good paths and by caching and processing data in the network. The directional information is utilized in this paradigm, and this protocol is mainly used in the observer-initiated application model [8]. However, it is necessary to design a routing algorithm in the continuous application model [8]. Therefore, this paper proposes a new multi-hop routing algorithm (WDM algorithm, Weight-Directed based Multi-hop routing algorithm) to meet the requirements of the continuous application model [8]. This approach can transfer data quickly to goal sensor node, i.e. sink node (See Fig. 1), using the directional information and RWVs of sensor nodes (RWV definition shown in Equation (2)), and balance energy consumption of all sensor nodes.

The remainder of this paper is organized as follows: The details of WDM algorithm are given in section 2 . The simulation results of analyses are illustrated in section 3. Finally, conclusions are made and some suggestions for future work are proposed.

\section{WDM Algorithm}

WSN is presented as undirected graph $G=(V, E)$, where $V$ is the set of all sensor nodes, i.e. $\mathrm{V}=\left\{\mathrm{V}_{1}, \mathrm{~V}_{2}, \ldots, \mathrm{V}_{\mathrm{n}}\right\}, \mathrm{N}=\{1,2, \ldots, \mathrm{n}\}$, and $\mathrm{E}$ is the set of edges in the network defined as follows:

$$
E=\left\{\left(V_{i}, V_{j}\right) \mid d\left(V_{i}, V_{j}\right) \leq R_{0}, V_{i}, V_{j} \in V, i, j \in N, i \neq j\right\}
$$

Where $d\left(V_{i}, V_{j}\right)$ is the distance between the neighbor nodes $V_{i}$ and $V_{j}, R_{0}$ is described as one-hop transmission range.

A number of definitions related to Equation (1) are given as follows:

(1) $V_{i}$.hop $p_{\min }$ is the minimal hops from sensor node $V_{i}$ to sink node, $V_{i} \in V$.

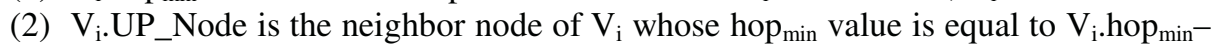
1. A set composed of these nodes is named as $\mathrm{V}_{\mathrm{i}}$.UpNodeSet. 
(3) $V_{i} \cdot$ Par_Node is the neighbor node of $V_{i}$ whose hop $p_{\min }$ value is equal to $V_{i}$.hop min $_{\text {min }}$. A set made up of these nodes is marked as $\mathrm{V}_{\mathrm{i}}$.ParNodeSet.

(4) $V_{i}$.Down_Node is the neighbor node of $V_{i}$ whose hop $p_{\min }$ value is equal to $\mathrm{V}_{\mathrm{i}} \cdot \mathrm{hop}_{\min }+1$. A set composed of these nodes is named as $\mathrm{V}_{\mathrm{i}}$. DownNodeSet.

(5) $\mathrm{V}_{\mathrm{i}}$.NBNodeSet is the union set of the above three sets.

(6) After system initialization, sink node first broadcasts route query packet to all sensor nodes. Then, each sensor node can gain route information, compute its hop $_{\min }$ value, and save its each neighbor node's hop $\mathrm{p}_{\min }$ and residual energy value $\mathrm{E}_{\mathrm{r}}$ in its cache.

In the case of invariable topology, once a source senor node $\mathrm{V}_{\mathrm{s}}$ senses a data packet of a particular mission, if a sensor node $V_{i}$ have gained the message and $\mathrm{V}_{\mathrm{s}}$. hop $\mathrm{p}_{\text {min }}$ value from node $\mathrm{V}_{\mathrm{s}}$, it will first compute route weight value (RWV) of its each neighbor nodes when choosing next hop node, which is defined as follows:

$$
V_{i} \cdot R W V\left(V_{k}\right)=\left(\frac{V_{s} \cdot h o p_{\min }}{V_{k} \cdot h o p_{\min }}\right)^{\alpha} \frac{V_{k} \cdot E_{r}}{V_{k} \cdot E_{0}}
$$

Where $\alpha$ is the effect factor of route direction, $V_{i} \in V, V_{k} \in V_{i} \cdot N B N o d e S e t, V_{k} \cdot E_{0}$ is the initial energy value of $\mathrm{V}_{\mathrm{k}}$. If each sensor node's initial energy is assumed as same value, $\mathrm{V}_{\mathrm{k}}$. $\mathrm{E}_{0}$ is abbreviated as $\mathrm{E}_{0}$.

Then, $\mathrm{V}_{\mathrm{i}}$ selects the neighbor node $\mathrm{V}_{\mathrm{k}}$ whose RWV is maximal, and also send the message and $\mathrm{V}_{\mathrm{s}}$. hop min $_{\text {malue to node }} \mathrm{V}_{\mathrm{k}}$. Subsequently, $\mathrm{V}_{\mathrm{k}}$ chooses the next hop node and transfers the packet to it until the message is sent to sink node.

When $V_{i}$ node has sent data packet to the node $V_{k}, V_{i}$ updates the remainder energy of its neighbor $\mathrm{V}_{\mathrm{k}}$. Specific computational approach is defined as follows:

$$
V_{k} \cdot E_{r}^{\prime}=V_{k} \cdot E_{r}-V_{k} \cdot E_{e x}-V_{k} \cdot E_{t x}
$$

Where $V_{k} \cdot E_{r}^{\prime}$ is the new residual energy value of node $\mathrm{V}_{\mathrm{k}} ; V_{k} \cdot E_{r}$ is the old residual energy value of node $\mathrm{V}_{\mathrm{k}} ; V_{k} \cdot E_{e x}$ is the energy consumption value when node $\mathrm{V}_{\mathrm{k}}$ receiving one data packet; $V_{k} \cdot E_{t x}$ is the energy consumption value when node $\mathrm{V}_{\mathrm{k}}$ transferring one data packet.

At the same time, $\mathrm{V}_{\mathrm{k}}$ also modifies the residual energy value of its neighbor $\mathrm{V}_{\mathrm{i}}$. The estimate method is described as follows:

$$
V_{i} \cdot E_{r}^{\prime}=V_{i} \cdot E_{r}-V_{i} \cdot E_{e x}-V_{i} \cdot E_{t x}-V_{i} \cdot E_{c}
$$

Where $V_{i} \cdot E_{c}$ is the energy consumption value of node $\mathrm{V}_{\mathrm{i}}$ computing and selecting next hop node; the meanings of $V_{i} \cdot E_{r}^{\prime}, V_{i} \cdot E_{r}, V_{i} . E_{e x}$, and $V_{i} \cdot E_{t x}$ are the same as above. 


\section{Performance Evaluation}

\subsection{Simulation Environment and Testing Criterion}

A square region of $100 \times 100 \mathrm{~m}^{2}$ has been generated and 160 sensor nodes are placed in the network randomly (See Fig. 1). All nodes start with an initial energy of 10J. The details of the sensor node's energy consumption model are shown in [9][10][11]. Data packet size is 525 bytes, $\alpha$ is equal to 1 , the maximum distance of one hop is $15 \mathrm{~m}$. It is assumed that a random node can sense one data packet of a particular mission at intervals of $1 \mathrm{~ms}$, and each message is finally routed to sink node.

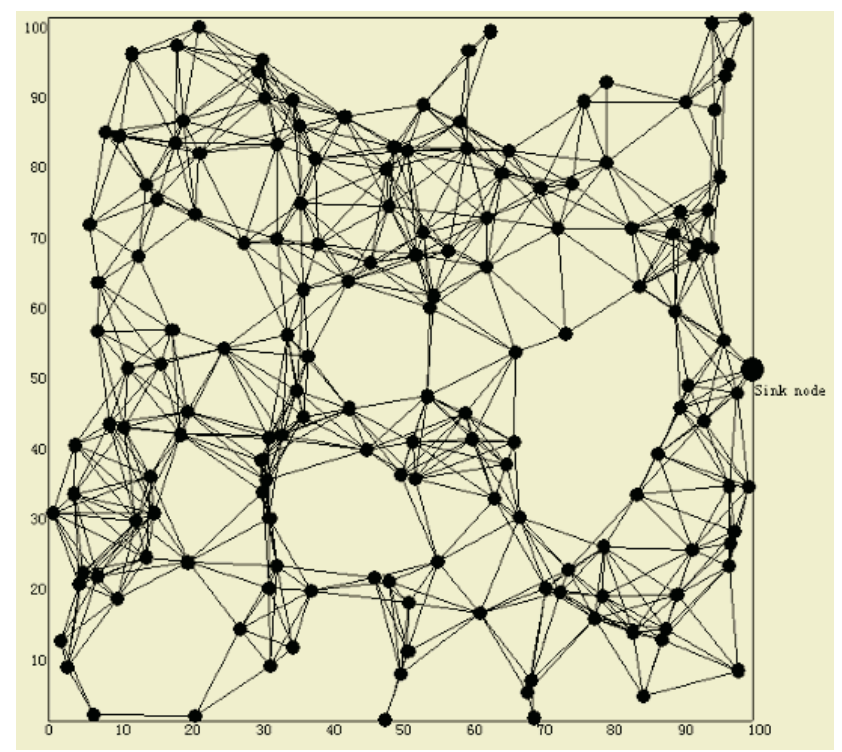

Fig. 1. Sensor nodes scattered in a wireless sensor network (sink node $(100,50)$ )

In order to analyze WDM algorithm performance, flooding algorithm is utilized to compare with WDM algorithm, and the following performance metrics are used:

(A) Average hops per data packet routed from source node to sink node (AHPD): this metric shows time delay of routing data and reflects whether it takes long time of algorithm to transfer data to sink node. A method of computing the metric is shown as follows:

$$
\text { AHPD }=\frac{\int_{0}^{t} \text { Data }_{-} \text {hops }(x) d x}{\int_{0}^{t} D_{\text {Data }} \text { num }(x) d x}
$$

Where Data_hops(t) is a linear function of hops with respect to time variable $t$; Data_num(t) is a linear function of data packet number about time variable $t$. 


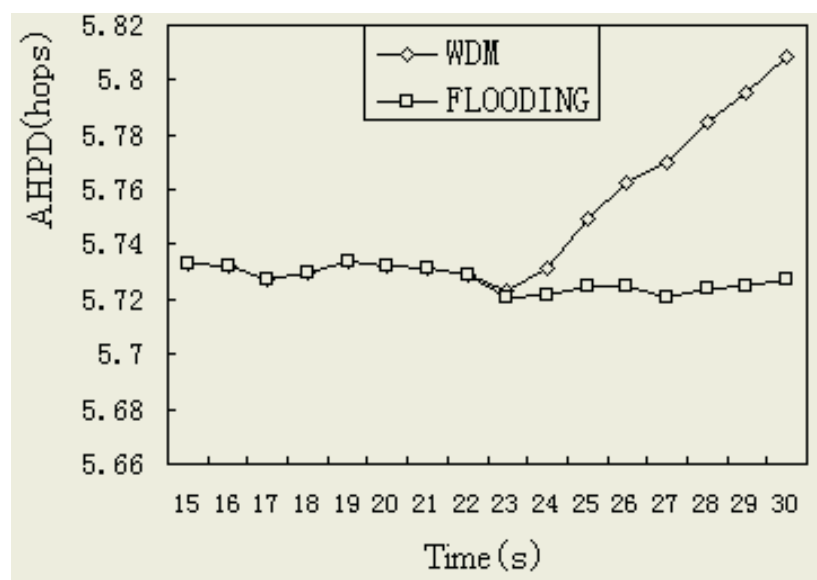

Fig. 2. Comparison of time delay of routing data between WDM and Flooding

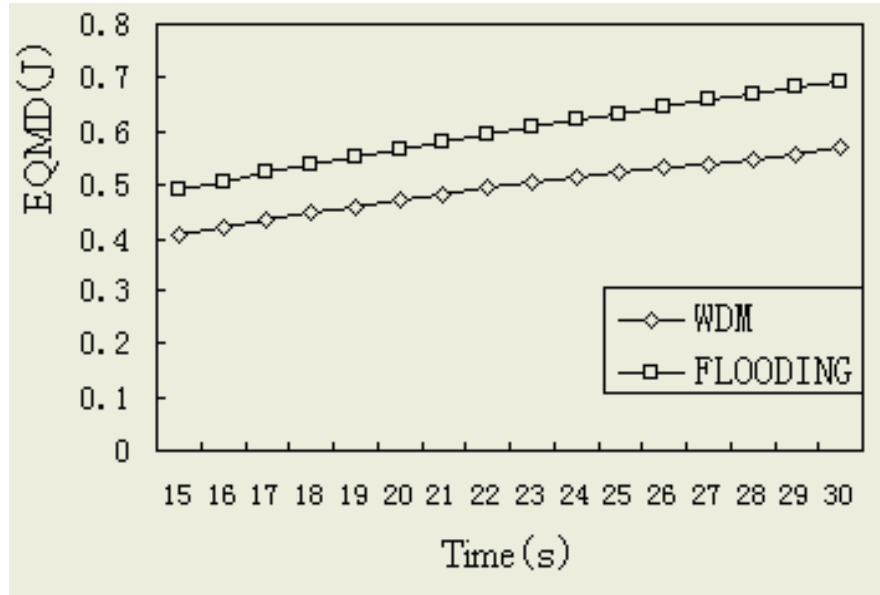

Fig. 3. Comparison of evenness of energy dissipated between WDM and Flooding

(B) Network energy quadratic mean deviation (EQMD): this metric indicates evenness of network energy dissipated of sensor nodes. A method of calculating this metric is listed as follows:

$$
E Q M D=\sqrt{\sum_{j}\left(\sum_{i} V_{i} \cdot E_{r} / A l l_{-} \text {nodes_Num- } V_{j} \cdot E_{r}\right)^{2}}
$$

Where All_nodes_Num is the total number of sensor nodes in the network; $\mathrm{V}_{\mathrm{i}} \cdot \mathrm{E}_{\mathrm{r}}$ is defined as residual energy value of one sensor node at the time.

(C) Average energy dissipated per data packet routed from source node to sink node (AEPD): this metric reflects energy costs of transferring data packets to sink node and shows energy-efficiency of algorithm. Combined with metric (B), it indicates the 
ability of algorithm extending system lifetime. A means of computing this metric is shown as follows:

$$
A E P D=\frac{\sum_{i} V_{i} \cdot E_{0}-\sum_{i} V_{i} \cdot E_{r}(t)}{\int_{0}^{t} D a t a_{-} n u m(x) d x}
$$

Where $V_{i} \cdot E_{r}(t)$ is the remainder energy value of node $V_{i}$ at one time $t$; the definitions of Vi.E $E_{0}$ and Data_num(t) are listed as above.

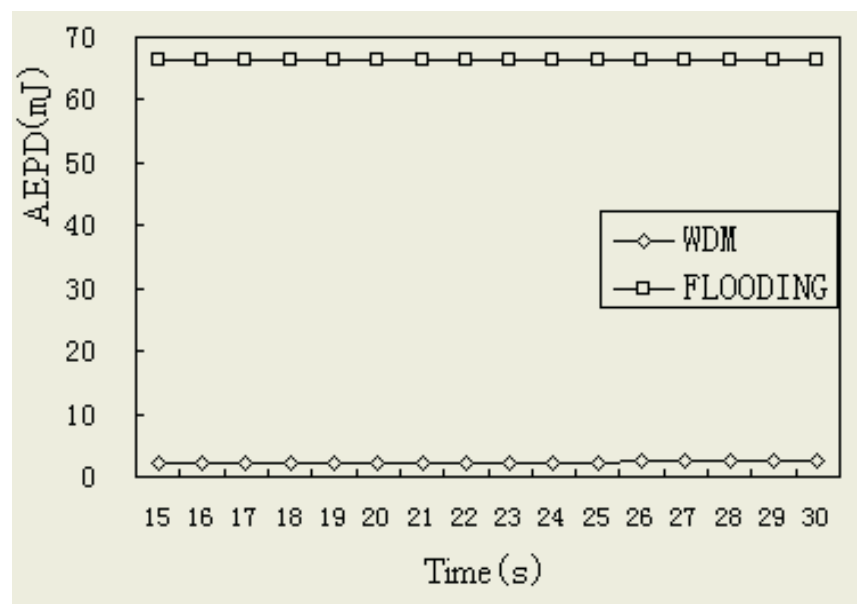

Fig. 4. Comparison of energy-efficiency between WDM and Flooding

\subsection{Result Discussion}

Firstly, in order to test time delay of routing data packets, simulation is performed with metric (A) and the results are shown in Fig. 2. It is found that the WDM algorithm can also transfer data quickly to sink node though it is slightly slower than the flooding algorithm because the latter is the quickest among all multi-hop routing algorithms for sensor networks.

Then, the evenness of dissipated network energy is also conducted with metric (B), as listed in Fig. 3. It is shown that two algorithms have the ability to balance energy consumption of all sensor nodes and the WDM algorithm is the better one.

Finally, comparison is made between WDM algorithm and flooding algorithm with metric $(\mathrm{C})$ to test energy efficiency, and the results are shown in Fig. 4. It is found that the flooding algorithm does pay much more energy costs to route one data packet and the WDM algorithm can gain better energy efficiency at all time. The average energy costs transferring one packet of the flooding algorithm is about 28.2 times of the WDM algorithm.

Hence, the results of the analyses above indicate that the WDM algorithm can gain quick data transmission, better evenness of dissipated network energy and energy efficiency to effectively extend network lifetime. 


\section{Conclusions}

In this paper, advantages and deficiencies of existing multi-hop routing algorithms are first analyzed. The WDM algorithm, an energy-efficient weight-directed based multihop algorithm, is proposed and described. The results of a series of simulations of sensor network environments indicate that the WDM algorithm can have the ability to transfer data quickly, balance network energy consumption of all sensor nodes, improve energy efficiency, and accordingly extend system lifetime. This algorithm is well suitable for the continuous model of static distributed WSNs. In the future topological transformation for several nodes death will be conducted so as to improve the algorithm to suit for dynamic distributed WSNs.

\section{Acknowledgments}

This work is supported by the National High-Tech Research and Development Plan of China under Grant No. 2003AA1Z2080.

\section{References}

1. Weiser, M.: The Computer for the 21st Century. Sci. Amer., Sept. (1991)

2. Zengwei, Zheng and Zhaohui, Wu: A Survey on Pervasive Computing. Computer Science, Vol. 30, No. 4. Chongqing, China, Apr. (2003) 18-22, 29

3. Ogawa M., Tamura, T., Togawa, T.: Fully automated biosignal acquisition in daily routine through 1 month. International Conference on IEEE-EMBS, Hong Kong, Oct. (1998)

4. Mainwaring, A., Polastre, J., Szewczyk, R. and Culler, D.: Wireless Sensor Networks for Habitat Monitoring. ACM WSNA'02, Atlanta, Georgia, Sept. (2002)

5. Intanagonwiwat C., Govindan R., Estrin D.: Directed diffusion: a scalable and robust communication paradigm for sensor networks. Proceedings of the ACM MobiCom'00, Boston, MA, Aug. (2000)

6. Estrin D., Govindan R., Heidemann J., Kumar S.: Next Century Challenges: Scalable Coordination in Sensor Networks. Proceedings of the ACM MobiCom'99, Seattle, Washington, Aug. (1999)

7. Hedetniemi S., Liestman A.: A survey of gossiping and broadcasting in communication networks. Networks, Vol. 18, No. 4, winter (1988) 319-349

8. Tilak S., Abu-Ghazaleh N., Heinzelman W.: A Taxonomy of Wireless Micro-Sensor Network Models. ACM Mobile Computing and Communications Review (MC2R), Vol. 6 , No. 2, Apr. (2002)

9. Zeng-wei Zheng, Zhao-hui Wu, Huai-zhong Lin: An Event-Driven Clustering Routing Algorithm for Wireless Sensor Networks. 2004 IEEE/RSJ International Conference on Intelligent Robots and Systems (IROS 2004), Sendai, Japan, Sept. (2004)

10. Sinha A., Chandrakasan A. P.: Energy Aware Software. Proceedings Of the 13th International Conference on VLSI Design, Calcutta, India, Jan. (2000)

11. Min R., Bhardwaj M., Cho S., Sinha A., et al.: An Architecture for a Power-Aware Distributed Microsensor Node. IEEE Workshop on Signal Processing Systems (SiPS ‘00) Design and Implementation, Lafayette, USA, Oct. (2000) 